\title{
Maximum Lateness Scheduling on Two-Person Cooperative Games with Variable Processing Times and Common Due Date
}

\author{
Peng Liu and Xiaoli Wang \\ School of Management, Shenyang University of Technology, Shenyang 110870, China \\ Correspondence should be addressed to Peng Liu; liup7802@163.com
}

Received 22 December 2016; Accepted 28 March 2017; Published 6 April 2017

Academic Editor: Manlio Gaudioso

Copyright ( 2017 Peng Liu and Xiaoli Wang. This is an open access article distributed under the Creative Commons Attribution License, which permits unrestricted use, distribution, and reproduction in any medium, provided the original work is properly cited.

\begin{abstract}
A new maximum lateness scheduling model in which both cooperative games and variable processing times exist simultaneously is considered in this paper. The job variable processing time is described by an increasing or a decreasing function dependent on the position of a job in the sequence. Two persons have to cooperate in order to process a set of jobs. Each of them has a single machine and their processing cost is defined as the minimum value of maximum lateness. All jobs have a common due date. The objective is to maximize the multiplication of their rational positive cooperative profits. A division of those jobs should be negotiated to yield a reasonable cooperative profit allocation scheme acceptable to them. We propose the sufficient and necessary conditions for the problems to have positive integer solution.
\end{abstract}

\section{Introduction}

In this paper, we introduce a new maximum lateness scheduling model in which both cooperative games and variable processing times exist simultaneously. There are many situations where one person is not able to undertake all the jobs alone in a large project and two persons need to cooperate in order to complete a project. Each person offers a single machine to process jobs. A division of those jobs should be negotiated to yield a reasonable cooperative profit allocation scheme acceptable to them. In many manufacturing processes, the processing times of jobs may be dependent on their positions in the sequence. This phenomenon is called "aging effect" or "learning effect" in the literature. In the aging effect, the processing time of a job increases as a function of its position in the sequence, while, in the learning effect, the processing time of a job decreases as a function of its position in the sequence. In this paper, we address maximum lateness scheduling on two-person cooperative games with variable processing times. To the best of our knowledge, no work has been done on maximum lateness scheduling models combining two aspects of cooperative games and variable processing times.
Scheduling problems with two-person cooperative games have received increasing attention in recent years. Jin et al. [1] consider the two-person cooperative games on makespan scheduling. The case of jobs with same processing time is addressed and each party's processing cost is determined by their minimized makespan. Dou et al. [2] consider the two-person cooperative games on total completion time scheduling where the processing time of each job is the same. $\mathrm{Gu}$ et al. [3] discuss the two-person Nash Bargaining problem with changeable processing time, where the processing cost is defined as their minimized maximum flow time. Jin [4] establishes a mathematical model of the problem where two persons process a batch of jobs by cooperation, where job processing time is a linear function of its starting time. Jin [5] studies two-person cooperative games problem on total completion time scheduling with changeable processing time. Gu et al. [6] consider the scheduling problem on two-person cooperative games, where the processing cost is defined as their minimized maximum latency. Liu et al. [7] study the problem of two-person cooperative games on minimizing the total (weighted) number of tardy jobs and on minimizing the total (weighted) completion time. Chen [8] studies an 
integer-valued cooperative games model on scheduling. Tang et al. [9] propose a new topic on dual relationships between job games and machine games and provide the extensive reviews of research on scheduling games. The literature mentioned above investigates the cooperative games on scheduling; however, they do not consider the maximum lateness scheduling with variable processing times.

Next, we will present a brief review of scheduling problems with variable processing times as follows. Gawiejnowicz [10] is the first time to introduce the position dependent on a number of executed jobs on a single processor. Biskup [11] is the first to consider the learning effect in a scheduling problem. Cheng and Wang [12] consider a single machine scheduling problem in which the job processing times will decrease as a result of learning. Mosheiov [13] proves that flow-time minimization with a learning effect on parallel identical machines has a polynomial time solution. Bachman and Janiak [14] investigate some single machine scheduling problems, where job processing times are defined by functions dependent on their positions in the sequence. Liu et al. [15] consider the single machine scheduling problems with two agents and position-dependent processing times. Rudek [16] provides the computational complexity results of scheduling problems with position-dependent job processing times, where the boundary between polynomially solvable and strongly NP-hard cases is given. J. B. Wang and J. J. Wang [17] investigate flowshop scheduling problems with a general exponential learning effect. Zhou and Zhang [18] consider the cooperative games based on multiple-machine scheduling problems with learning effects. Zhang et al. [19] propose a group scheduling model with deteriorating and learning effect on a single machine. The aging effect is mentioned for the first time by Mosheiov [13] during analysis of the learning effect. Janiak and Rudek [20] prove that the makespan minimization problems on a single machine with release dates and some special cases of the given aging effect are NP-hard. Yang et al. [21] consider single machine scheduling and slack due date assignment problems simultaneously with the position-dependent aging effect and deteriorating maintenance. Rudek [22] analyzes the single machine maximum lateness minimization scheduling problem with processing time-based aging effects. Ji et al. [23] consider a single machine due date assignment scheduling problem with jobdependent aging effects and a deteriorating maintenance activity. Choi [24] considers a two-agent single machine scheduling problem with linear position-based aging effects and job-dependent aging ratios. Zeng et al. [25] consider the problem of bidirectional nonnegative deep model and its optimization in learning. The difference between our model and the above scheduling models is that we incorporate the important factor of cooperative games in the model.

The remainder of this paper is organized as follows. In Section 2, we describe the proposed problems. In Section 3, we discuss the two-person cooperative games on maximum lateness scheduling problems with an aging effect and a learning effect, respectively. Section 4 gives some concluding remarks.

\section{Problem Description}

The problems of maximum lateness scheduling on twoperson cooperative games with variable processing times are described as follows. There is a set of jobs $J=\left\{J_{1}, J_{2}, \ldots, J_{n}\right\}$ that has to be processed by two persons. Each job should be processed only once without interruption. All jobs are available for processing at time zero. Two persons, each with a single machine, cooperate to complete the processing of these $n$ jobs. The due date of each job is denoted by $d_{j}$, and all jobs have a common due date $d$; that is, $d_{j}=d$. The processing time $p_{j, r}$ of job $J_{j}$ is a function dependent on its position $r$ in a sequence. We consider two special models of job variable processing times: (1) an aging effect $p_{j, r}=P_{0}+$ ar and (2) a learning effect $p_{j, r}=P_{0}-a r, j=1,2, \ldots, n, r=1,2, \ldots, n$, where $P_{0}$ is the normal processing time, $a>0$ denotes a constant aging ratio in model (1) and a constant learning ratio in model (2). Since job processing time is positive value, it is assumed that $a<P_{0} / n$ for the learning effect model. The normal processing time and the aging/learning ratio are identical for all jobs.

Given a solution for the two-person cooperative games, we use $C_{j}$ to denote the completion time for job $J_{j}$. If we use $X_{1}$ and $X_{2}$ to denote each person's subset of jobs, then $X_{1} \cup X_{2}=J, X_{1} \cap X_{2}=\varnothing$. For person $i(i=1,2)$, processing a job per unit time will bring $b_{i}(i=1,2)$ unit profit; the processing cost is defined as the minimum value of the maximum lateness of jobs, that is, $\min L_{\max }^{i}=\min \max \left\{C_{j}-d \mid\right.$ $j \in X_{i}$ \}, and then the profit function of person $i$ is defined by $u_{i}=b_{i} \sum_{j \in X_{i}} p_{j, r}-\min L_{\max }^{i}$. If two persons cannot achieve a cooperation agreement, let $e_{i}$ denote the $i$ th individual's cost; then there should be the inequalities of the form $e_{i} \geq$ $u_{i}(\phi)=0,(i=1,2)$. Hence, the cooperative profit of person $i$ is formulated by $v_{i}=u_{i}\left(X_{i}\right)-e_{i}=b_{i} \sum_{j \in X_{i}} p_{j, r}-\min L_{\max }^{i}-e_{i}$, the overall profit of two persons is measured by the product of $v_{1}$ and $v_{2}$, and our objective is to maximize this product.

The problems of maximum lateness scheduling on twoperson cooperative games with variable processing times and common due date can be described by the three-field notation of Jin et al. [1] of the forms G2| $p_{j, r}=P_{0}+a r, d_{j}=d \mid v_{1} v_{2} / L_{\max }$ and $G 2\left|p_{j, r}=P_{0}-a r, d_{j}=d\right| v_{1} v_{2} / L_{\max }$, respectively. The first-field $G 2$ represents two-person cooperative games and each person has one machine. The second field describes job characteristics, that is, the structures of the processing time and the due date. The third field $v_{1} v_{2} / L_{\max }$ represents the optimization goal.

\section{Main Results}

We consider the two-person cooperative games on maximum lateness scheduling problems $G 2\left|p_{j, r}=P_{0}+a r, d_{j}=d\right| v_{1} v_{2} \mid$ $L_{\max }$ and $G 2\left|p_{j, r}=P_{0}-a r, d_{j}=d\right| v_{1} v_{2} / L_{\max }$ in Sections 3.1 and 3.2 , respectively.

3.1. Problem G2 $\left|p_{j, r}=P_{0}+a r, d_{j}=d\right| v_{1} v_{2} / L_{\max }$. Let $k$ be the number of jobs in subset $X_{1}$ assigned to person 1 , and let $n-k$ be the number of jobs in subset $X_{2}$ assigned to person 2, 
$1 \leq k \leq n-1$. Next, we will show that $C_{k}=k P_{0}+(k(k+1) / 2) a$ for person 1 by induction.

$$
\begin{aligned}
C_{1} & =p_{1,1}=P_{0}+a=P_{0}+\frac{1 \times(1+1)}{2} a . \\
C_{2} & =C_{1}+p_{2,2}=\left(P_{0}+a\right)+\left(P_{0}+2 a\right) \\
& =2 P_{0}+\frac{2 \times(2+1)}{2} a .
\end{aligned}
$$

Suppose $C_{j}=j P_{0}+(j(j+1) / 2) a$ for job $J_{j}$; then we consider job $J_{j+1}$ :

$$
\begin{aligned}
C_{j+1} & =C_{j}+P_{(j+1),(j+1)} \\
& =j P_{0}+\frac{j(j+1)}{2} a+\left(P_{0}+a(j+1)\right) \\
& =(j+1) P_{0}+\frac{(j+1)(j+2)}{2} a .
\end{aligned}
$$

Hence, we prove $C_{k}=k P_{0}+(k(k+1) / 2) a$, which is a fixed constant for all permutations of the jobs.

Likewise, we can show that $C_{n}=(n-k) P_{0}+((n-k)(n-$ $k+1) / 2) a$ for person 2 by induction. $C_{n}=(n-k) P_{0}+(1+$ $2+\cdots+n-k) a=(n-k) P_{0}+((n-k)(n-k+1) / 2) a$, which is a fixed constant for all permutations of the jobs.

$$
\begin{aligned}
& L_{\text {max }}^{1}=\max \left\{C_{j}-d_{j} \mid j \in X_{1}\right\}=\max \left\{j P_{0}\right. \\
& \left.\quad+\frac{j(j+1)}{2} a-d_{j} \mid j \in X_{1}\right\}=\max \left\{P_{0}+a-d, 2 P_{0}\right. \\
& \left.\quad+\frac{2 \times(2+1)}{2} a-d, \ldots, k P_{0}+\frac{k(k+1)}{2} a-d\right\} \\
& \quad=k P_{0}+\frac{k(k+1)}{2} a-d, \\
& L_{\text {max }}^{2}=\max \left\{C_{j}-d_{j} \mid j \in X_{2}\right\}=(n-k) P_{0} \\
& \quad+\frac{(n-k)(n-k+1)}{2} a-d .
\end{aligned}
$$

The profit functions are as follows:

$$
\begin{aligned}
u_{1}\left(X_{1}\right) & =b_{1} \sum_{j \in X_{1}} p_{j, r}-\min L_{\max }^{1}=b_{1} C_{k}-\min L_{\max }^{1} \\
= & \left(b_{1}-1\right)\left[k P_{0}+\frac{k(k+1)}{2} a\right]+d, \\
u_{2}\left(X_{2}\right)= & b_{2} \sum_{j \in X_{2}} p_{j, r}-\min L_{\max }^{2} \\
= & \left(b_{2}-1\right)\left[(n-k) P_{0}+\frac{(n-k)(n-k+1)}{2} a\right] \\
& +d .
\end{aligned}
$$

Since the profit functions have positive values, it should hold that $b_{i}>1,(i=1,2)$.
Thus, the two cooperative profit functions are the quadratic functions of positive integer $k$ of the form

$$
\begin{aligned}
& v_{1}(k)=u_{1}\left(X_{1}\right)-e_{1}=\left(b_{1}-1\right)\left[k P_{0}+\frac{k(k+1)}{2} a\right] \\
& +d-e_{1}=\frac{a\left(b_{1}-1\right)}{2}\left[k^{2}+\left(1+\frac{2 P_{0}}{a}\right) k\right. \\
& \left.\quad+\frac{2\left(d-e_{1}\right)}{a\left(b_{1}-1\right)}\right], \\
& v_{2}(k)=u_{2}\left(X_{2}\right)-e_{2}=\left(b_{2}-1\right)\left[(n-k) P_{0}\right. \\
& \left.+\frac{(n-k)(n-k+1)}{2} a\right]+d-e_{2}=\frac{a\left(b_{2}-1\right)}{2}\left\{k^{2}\right. \\
& \quad-\left(1+2 n+\frac{2 P_{0}}{a}\right) k \\
& \left.+\left[n^{2}+n+\frac{2 n P_{0}}{a}+\frac{2\left(d-e_{2}\right)}{a\left(b_{2}-1\right)}\right]\right\} .
\end{aligned}
$$

When the discriminant $\Delta_{1}=\left(1+2 P_{0} / a\right)^{2}-8\left(d-e_{1}\right) / a\left(b_{1}-\right.$ 1) of $v_{1}$ and the discriminant $\Delta_{2}=\left(2 P_{0} / a+1\right)^{2}-8(d-$ $\left.e_{2}\right) / a\left(b_{2}-1\right)$ of $v_{2}$ are smaller than zero, we do not need to study this problem. So we only consider the solution when

$$
\begin{aligned}
\Delta_{i} & \geq 0, \\
e_{i} & \geq 0, \\
b_{i} & >1, \quad(i=1,2), \\
a & >0, \\
1 & \leq k \leq n-1 .
\end{aligned}
$$

Under the condition of $\Delta_{i} \geq 0, v_{1}(k) v_{2}(k)$ can be expressed as the following form:

$$
\begin{aligned}
v_{1}(k) v_{2}(k)= & \frac{a^{2}\left(b_{1}-1\right)\left(b_{2}-1\right)}{4}\left(k-\alpha_{1}\right)\left(k-\alpha_{2}\right) \\
& \cdot\left(k-\alpha_{3}\right)\left(k-\alpha_{4}\right),
\end{aligned}
$$

where

$$
\begin{aligned}
& \alpha_{1}=-\left(\frac{1}{2}+\frac{P_{0}}{a}\right)-\sqrt{\left(\frac{1}{2}+\frac{P_{0}}{a}\right)^{2}-\frac{2\left(d-e_{1}\right)}{a\left(b_{1}-1\right)}}, \\
& \alpha_{2}=-\left(\frac{1}{2}+\frac{P_{0}}{a}\right)+\sqrt{\left(\frac{1}{2}+\frac{P_{0}}{a}\right)^{2}-\frac{2\left(d-e_{1}\right)}{a\left(b_{1}-1\right)}}, \\
& \alpha_{3}=n+\frac{1}{2}+\frac{P_{0}}{a}-\sqrt{\left(\frac{1}{2}+\frac{P_{0}}{a}\right)^{2}-\frac{2\left(d-e_{2}\right)}{a\left(b_{2}-1\right)}}, \\
& \alpha_{4}=n+\frac{1}{2}+\frac{P_{0}}{a}+\sqrt{\left(\frac{1}{2}+\frac{P_{0}}{a}\right)^{2}-\frac{2\left(d-e_{2}\right)}{a\left(b_{2}-1\right)}} .
\end{aligned}
$$


Theorem 1. The sufficient and necessary condition for the problem $G 2\left|p_{j, r}=P_{0}+a r, d_{j}=d\right| v_{1} v_{2} / L_{\max }$ to have positive integer solution $k$ is

$$
\beta_{1} \leq \beta_{2} .
$$

Furthermore, if the problem has a solution, then the optimal solution $k^{*}$ is certainly in closed interval $\left[\beta_{1}, \beta_{2}\right]$, where $\beta_{1}=$ $\left\lfloor\alpha_{2}\right\rfloor+1, \beta_{2}=\left\lceil\alpha_{3}\right\rceil-1$.

Proof. If the problem has a solution, the two cooperative profit functions $v_{1}$ and $v_{2}$ must be of positive value. We have $k<\alpha_{1}$ and $k>\alpha_{2}$ from $v_{1}>0$. We obtain $k<\alpha_{3}$ and $k>\alpha_{4}$ from $v_{2}>0$. Since $\alpha_{1}<0, \alpha_{4}>n$, and $1 \leq k \leq n-1$, we have $\alpha_{2}<k<\alpha_{3}$. Hence, we have $k \in\left(\alpha_{2}, \alpha_{3}\right) \cap[1, n-1]$. Since $k$ is a integer, we set $\beta_{1}=\left\lfloor\alpha_{2}\right\rfloor+1, \beta_{2}=\left\lceil\alpha_{3}\right\rceil-1$. Thus, $1 \leq \beta_{1} \leq n-1,1 \leq \beta_{2} \leq n-1$. So the problem $G 2 \mid p_{j, r}=$ $P_{0}+a r, d_{j}=d \mid v_{1} v_{2} / L_{\max }$ to have a solution equivalent to the closed interval $\left[\beta_{1}, \beta_{2}\right]$ has at least one positive integer $k$, that is, $\beta_{1} \leq \beta_{2}$. Therefore, the sufficient and necessary condition for the problem $G 2\left|p_{j, r}=P_{0}+a r, d_{j}=d\right| v_{1} v_{2} / L_{\max }$ to have positive integer solution $k$ is $\beta_{1} \leq \beta_{2}$.

We set $v_{1}(x) \cdot v_{2}(x)$ as a four-time polynomial in $x$ and the function can be expressed as $v_{1}(x) \cdot v_{2}(x)=\left(a^{2}\left(b_{1}-1\right)\left(b_{2}-\right.\right.$ 1)/4) $\left(x-\alpha_{1}\right)\left(x-\alpha_{2}\right)\left(x-\alpha_{3}\right)\left(x-\alpha_{4}\right)$. So $\left[v_{1}(x) \cdot v_{2}(x)\right]^{\prime}=0$ is a cubic polynomial in $x$ and $\left[v_{1}(x) \cdot v_{2}(x)\right]^{\prime}=0$ have three roots. Under the conditions of (6) and (9), we know that there are three roots to $\left[v_{1}(k) \cdot v_{2}(k)\right]^{\prime}=0$. Without loss of generality, let us set them to be $\delta_{1}, \delta_{2}, \delta_{3}$ and their relationship can be defined as $\delta_{1}<\delta_{2}<\delta_{3}$. So $\delta_{1}, \delta_{2}$, and $\delta_{3}$ are the three extreme points of $v_{1}(k) \cdot v_{2}(k)$ and we can get $\beta_{1} \leq \delta_{2} \leq \beta_{2}$. Since the coefficient of four times polynomial about expression (7) is positive, the coefficient of cubic polynomial $\left[v_{1}(k) \cdot v_{2}(k)\right]^{\prime}$ is positive and $\delta_{2}$ is the only extreme point and maximum value point. Therefore, the optimal solution $k^{*}$ of the problem $G 2\left|p_{j, r}=P_{0}+a r, d_{j}=d\right| v_{1} v_{2} / L_{\max }$ is certainly in closed interval $\left[\beta_{1}, \beta_{2}\right]$.

We have the following theorem from Theorem 1.

Theorem 2. Under the condition of expression (9), there is an optimal solution $k^{*}$ for the problem $G 2 \mid p_{j, r}=P_{0}+a r, d_{j}=$ $d \mid v_{1} v_{2} / L_{\max }$, and the result can be expressed as $k^{*}=\left\lfloor\delta_{2}\right\rfloor$ or $\left\lceil\delta_{2}\right\rceil$. The optimal solution $k^{*}$ is as follows:

(I) If $\left\lceil\delta_{2}\right\rceil \in\left[\beta_{1}, \beta_{2}\right]$ and $\left\lfloor\delta_{2}\right\rfloor \notin\left[\beta_{1}, \beta_{2}\right]$, then $k^{*}=\left\lceil\delta_{2}\right\rceil$;

(II) If $\left\lfloor\delta_{2}\right\rfloor \in\left[\beta_{1}, \beta_{2}\right]$ and $\left\lceil\delta_{2}\right] \notin\left[\beta_{1}, \beta_{2}\right]$, then $k^{*}=\left\lfloor\delta_{2}\right\rfloor$;

(III) If $\left\lceil\delta_{2}\right\rceil \in\left[\beta_{1}, \beta_{2}\right]$ and $\left\lfloor\delta_{2}\right\rfloor \in\left[\beta_{1}, \beta_{2}\right]$,

$$
\begin{aligned}
& \text { when } v_{1}\left(\left\lfloor\delta_{2}\right\rfloor\right) \cdot v_{2}\left(\left\lfloor\delta_{2}\right\rfloor\right) \leq v_{1}\left(\left\lceil\delta_{2}\right\rceil\right) \cdot v_{2}\left(\left\lceil\delta_{2}\right\rceil\right), \\
& k^{*}=\left\lceil\delta_{2}\right\rceil ; \\
& \text { when } v_{1}\left(\left\lfloor\delta_{2}\right\rfloor\right) \cdot v_{2}\left(\left\lfloor\delta_{2}\right\rfloor\right) \geq v_{1}\left(\left\lceil\delta_{2}\right\rceil\right) \cdot v_{2}\left(\left\lceil\delta_{2}\right\rceil\right), \\
& k^{*}=\left\lfloor\delta_{2}\right\rfloor .
\end{aligned}
$$

Remark 3. The problem size is $O(1)$ and the optimal solution can also be found in a constant time $O(1)$.

Furthermore, we consider the following numerical example.
Example 4. For the problem G2 $\left|p_{j, r}=P_{0}+a r, d_{j}=d\right| v_{1} v_{2} \mid$ $L_{\max }$, we assume that $P_{0}=1, a=1, d=11, b_{1}=2, b_{2}=3$, $e_{1}=11, e_{2}=10$, and $n=5$.

Solution. The two cooperative profit functions are

$$
\begin{aligned}
& v_{1}(k)=\frac{a\left(b_{1}-1\right)}{2}\left[k^{2}+\left(1+\frac{2 P_{0}}{a}\right) k+\frac{2\left(d-e_{1}\right)}{a\left(b_{1}-1\right)}\right] \\
& =\frac{1}{2} k^{2}+\frac{3}{2} k, \\
& v_{2}(k)=\frac{a\left(b_{2}-1\right)}{2}\left\{k^{2}-\left(1+2 n+\frac{2 P_{0}}{a}\right) k\right. \\
& \left.\quad+\left[n^{2}+n+\frac{2 n P_{0}}{a}+\frac{2\left(d-e_{2}\right)}{a\left(b_{2}-1\right)}\right]\right\}=k^{2}-13 k \\
& \quad+41 .
\end{aligned}
$$

We have $\Delta_{1}=9 / 4>0, \Delta_{2}=5>0, \alpha_{1}=-3, \alpha_{2}=0$, $\alpha_{3}=5.382$, and $\alpha_{4}=7.618$. So we obtain $\beta_{1}=\left\lfloor\alpha_{2}\right\rfloor+1=1$, $\beta_{2}=\left\lceil\alpha_{3}\right\rceil-1=5$. Three roots to $\left[v_{1}(k) \cdot v_{2}(k)\right]^{\prime}=0$ are $\delta_{1}=-1.768, \delta_{2}=2.613$, and $\delta_{3}=6.656$, respectively. So we obtain $k^{*}=\left\lfloor\delta_{2}\right\rfloor=2$ or $k^{*}=\left\lceil\delta_{2}\right\rceil=3$. Because $v_{1}(2) \cdot v_{2}(2)=$ $94.978<v_{1}(3) \cdot v_{2}(3)=98.9604$, we have $k^{*}=3$.

3.2. Problem $G 2\left|p_{j, r}=P_{0}-a r, d_{j}=d\right| v_{1} v_{2} / L_{\max }$. For the problem $G 2\left|p_{j, r}=P_{0}-a r, d_{j}=d\right| v_{1} v_{2} / L_{\max }$, we can calculate similarly the makespan for persons 1 and 2 by induction.

$C_{k}=k P_{0}-(k(k+1) / 2) a$, which is a fixed constant for all permutations of the jobs.

$C_{n}=(n-k) P_{0}-((n-k)(n-k+1) / 2) a$, which is a fixed constant for all permutations of the jobs.

$$
\begin{aligned}
& L_{\text {max }}^{1}=\max \left\{C_{j}-d_{j} \mid j \in X_{1}\right\}=\max \left\{j P_{0}\right. \\
& \left.-\frac{j(j+1)}{2} a-d_{j} \mid j \in X_{1}\right\}=\max \left\{P_{0}-a\right. \\
& -d, 2 P_{0}-\frac{2 \times(2+1)}{2} a-d, \ldots, k P_{0}-\frac{k(k+1)}{2} a \\
& -d\}=k P_{0}-\frac{k(k+1)}{2} a-d, \\
& L^{2}{ }_{\max }=\max \left\{C_{j}-d_{j} \mid j \in X_{2}\right\}=(n-k) P_{0} \\
& -\frac{(n-k)(n-k+1)}{2} a-d .
\end{aligned}
$$

The profit functions are as follows.

$$
\begin{gathered}
u_{1}\left(X_{1}\right)=b_{1} \sum_{j \in X_{1}} p_{j, r}-\min L_{\max }^{1}=b_{1} C_{k}-\min L_{\max }^{1} \\
=\left(b_{1}-1\right)\left[k P_{0}-\frac{k(k+1)}{2} a\right]+d,
\end{gathered}
$$




$$
\begin{aligned}
& u_{2}\left(X_{2}\right)=b_{2} \sum_{j \in X_{2}} p_{j, r}-\min L_{\max }^{2} \\
& \quad=\left(b_{2}-1\right)\left[(n-k) P_{0}-\frac{(n-k)(n-k+1)}{2} a\right]+d .
\end{aligned}
$$

Since the profit functions have positive values, it should hold that $b_{i}>1,(i=1,2)$.

Thus, the two cooperative profit functions are the quadratic functions of positive integer $k$ of the form

$$
\begin{aligned}
& v_{1}(k)=u_{1}\left(X_{1}\right)-e_{1}=\left(b_{1}-1\right)\left[k P_{0}-\frac{k(k+1)}{2} a\right] \\
& +d-e_{1}=-\frac{a\left(b_{1}-1\right)}{2}\left[k^{2}-\left(\frac{2 P_{0}}{a}-1\right) k\right. \\
& \left.\quad-\frac{2\left(d-e_{1}\right)}{a\left(b_{1}-1\right)}\right], \\
& v_{2}(k)=u_{2}\left(X_{2}\right)-e_{2}=\left(b_{2}-1\right)\left[(n-k) P_{0}\right. \\
& \left.-\frac{(n-k)(n-k+1)}{2} a\right]+d-e_{2} \\
& =-\frac{a\left(b_{2}-1\right)}{2}\left\{k^{2}-\left(1+2 n-\frac{2 P_{0}}{a}\right) k\right. \\
& \left.+\left[n^{2}+n-\frac{2 n P_{0}}{a}-\frac{2\left(d-e_{2}\right)}{a\left(b_{2}-1\right)}\right]\right\} .
\end{aligned}
$$

Since the profit functions have positive values, it should hold that $b_{i}>1,(i=1,2)$.

When the discriminant $\Delta_{1}=\left(2 P_{0} / a-1\right)^{2}+8\left(d-e_{1}\right) / a\left(b_{1}-\right.$ 1) of $v_{1}$ and the discriminant $\Delta_{2}=\left(2 P_{0} / a-1\right)^{2}+8(d-$ $\left.e_{2}\right) / a\left(b_{2}-1\right)$ of $v_{2}$ are smaller than zero, we do not need to study this problem. So we only consider the solution when

$$
\begin{aligned}
& \Delta_{i} \geq 0, \\
& e_{i} \geq 0, \\
& b_{i}>1, \quad(i=1,2), \\
& 0<a<\frac{P_{0}}{n}, \\
& 1 \leq k \leq n-1 .
\end{aligned}
$$

Under the condition of $\Delta_{i} \geq 0, v_{1}(k) v_{2}(k)$ can be expressed as the following form:

$$
\begin{aligned}
v_{1}(k) v_{2}(k)= & \frac{a^{2}\left(b_{1}-1\right)\left(b_{2}-1\right)}{4}\left(k-\gamma_{1}\right)\left(k-\gamma_{2}\right) \\
& \cdot\left(k-\gamma_{3}\right)\left(k-\gamma_{4}\right),
\end{aligned}
$$

where

$$
\begin{aligned}
& \gamma_{1}=\frac{P_{0}}{a}-\frac{1}{2}-\sqrt{\left(\frac{P_{0}}{a}-\frac{1}{2}\right)^{2}+\frac{2\left(d-e_{1}\right)}{a\left(b_{1}-1\right)}}, \\
& \gamma_{2}=\frac{P_{0}}{a}-\frac{1}{2}+\sqrt{\left(\frac{P_{0}}{a}-\frac{1}{2}\right)^{2}+\frac{2\left(d-e_{1}\right)}{a\left(b_{1}-1\right)}}, \\
& \gamma_{3}=n+\frac{1}{2}-\frac{P_{0}}{a}-\sqrt{\left(\frac{P_{0}}{a}-\frac{1}{2}\right)^{2}+\frac{2\left(d-e_{2}\right)}{a\left(b_{2}-1\right)}}, \\
& \gamma_{4}=n+\frac{1}{2}-\frac{P_{0}}{a}+\sqrt{\left(\frac{P_{0}}{a}-\frac{1}{2}\right)^{2}+\frac{2\left(d-e_{2}\right)}{a\left(b_{2}-1\right)}} .
\end{aligned}
$$

In consideration of clear expression, we set $\gamma_{5}=P_{0} / a-$ $1 / 2, \gamma_{6}=n+1 / 2-P_{0} / a$.

Theorem 5. The sufficient and necessary condition for the problem $G 2\left|p_{j, r}=P_{0}-a r, d_{j}=d\right| v_{1} v_{2} / L_{\max }$ to have positive integer solution $k$ is

$$
\eta_{l} \leq \eta_{m}
$$

Furthermore, if the problem has a solution, then the optimal solution $k^{*}$ is certainly in closed interval $\left[\eta_{l}, \eta_{m}\right]$, where $\eta_{l}=$ $\max \left\{\eta_{l 1}, \eta_{l 2}\right\}, \eta_{m}=\min \left\{\eta_{m 1}, \eta_{m 2}\right\}, \eta_{l 1}=\left\lfloor\gamma_{1}\right\rfloor+1, \eta_{l 2}=\left\lfloor\gamma_{6}\right\rfloor+1$, $\eta_{m 1}=\left\lceil\gamma_{5}\right\rceil-1$, and $\eta_{m 2}=\left\lceil\gamma_{4}\right\rceil-1$.

Proof. If the problem has a solution, the two cooperative profit functions $v_{1}$ and $v_{2}$ must be of positive value. We have $\gamma_{1}<k<\gamma_{2}$ from $v_{1}>0$. Since $0<a<P_{0} / n$, we have $k<P_{0} / a-1 / 2$; that is, $k<\gamma_{5}$. Then, we know $\gamma_{5}<\gamma_{2}$ through the comparing between $\gamma_{2}$ and $\gamma_{5}$. So we have $\gamma_{1}<k<\gamma_{5}$.

We get $\gamma_{3}<k<\gamma_{4}$ from $v_{2}>0$. Because $k-1 / 2-n+P_{0} / a>$ 0 , so we have $k>\gamma_{6}$. Since $\gamma_{3}<\gamma_{6}$, then we have $\gamma_{6}<k<\gamma_{4}$.

Thus, we obtain $\gamma_{1}<k<\gamma_{5}$ and $\gamma_{6}<k<\gamma_{4}$. Since $1 \leq k \leq n-1$ and $k$ is a integer, so we set $\eta_{l 1}=\left\lfloor\gamma_{1}\right\rfloor+1$, $\eta_{l 2}=\left\lfloor\gamma_{6}\right\rfloor+1, \eta_{m 1}=\left\lceil\gamma_{5}\right\rceil-1, \eta_{m 2}=\left\lceil\gamma_{4}\right\rceil-1, \eta_{l}=\max \left\{\eta_{l 1}, \eta_{l 2}\right\}$, and $\eta_{m}=\min \left\{\eta_{m 1}, \eta_{m 2}\right\}$. Then we obtain $0 \leq \gamma_{1}<n-1$, $1 \leq \gamma_{4}<n, 1 \leq \gamma_{5}<n, 1 \leq \gamma_{6}<n$, and $n \geq 2$ from the expressions of $\gamma_{1}, \gamma_{4}, \gamma_{5}$, and $\gamma_{6}$. Hence, $1 \leq \eta_{l} \leq n-1$, $1 \leq \eta_{m} \leq n-1$. Therefore, the sufficient and necessary condition for the problem $G 2\left|p_{j, r}=P_{0}-a r, d_{j}=d\right| v_{1} v_{2} / L_{\text {max }}$ to have integer solution $k$ is $\eta_{l} \leq \eta_{m}$. Similarly, we can prove that the optimal solution $k^{*}$ is certainly in closed interval $\left[\eta_{l}, \eta_{m}\right]$.

We have the following Theorem 6 that is similar to Theorem 2. If we prove $\xi_{2} \in\left[\eta_{l}, \eta_{m}\right]$, we obtain that $\xi_{2}$ is the only extreme point of $v_{1}(x) \cdot v_{2}(x)$ in $\left[\eta_{l}, \eta_{m}\right]$ and it is also the maximum value point of $v_{1}(x) \cdot v_{2}(x)$.

Theorem 6. Under the condition of expression (17), there is an optimal solution $k^{*}$ for the problem $G 2 \mid p_{j, r}=P_{0}-$ ar, $d_{j}=$ $d \mid v_{1} v_{2} / L_{\max }$, and the result can be expressed as $k^{*}=\left\lfloor\xi_{2}\right\rfloor$ or $\left\lceil\xi_{2}\right\rceil$. The optimal solution $k^{*}$ is as follows:

(I) If $\left\lceil\xi_{2}\right\rceil \in\left[\eta_{l}, \eta_{m}\right]$ and $\left\lfloor\xi_{2}\right\rfloor \notin\left[\eta_{l}, \eta_{m}\right]$, then $k^{*}=\left\lceil\xi_{2}\right\rceil$;

(II) If $\left\lfloor\xi_{2}\right\rfloor \in\left[\eta_{l}, \eta_{m}\right]$ and $\left\lceil\xi_{2}\right\rceil \notin\left[\eta_{l}, \eta_{m}\right]$, then $k^{*}=\left\lfloor\xi_{2}\right\rfloor$; 
(III) If $\left\lceil\xi_{2}\right\rceil \in\left[\eta_{l}, \eta_{m}\right]$ and $\left\lfloor\xi_{2}\right\rfloor \in\left[\eta_{l}, \eta_{m}\right]$,

$$
\begin{aligned}
& \text { when } v_{1}\left(\left\lfloor\xi_{2}\right\rfloor\right) \cdot v_{2}\left(\left\lfloor\xi_{2}\right\rfloor\right) \leq v_{1}\left(\left\lceil\xi_{2}\right\rceil\right) \cdot v_{2}\left(\left\lceil\xi_{2}\right\rceil\right) \text {, then } \\
& k^{*}=\left\lceil\xi_{2}\right\rceil ; \\
& \text { when } v_{1}\left(\left\lfloor\xi_{2}\right\rfloor\right) \cdot v_{2}\left(\left\lfloor\xi_{2}\right\rfloor\right) \geq v_{1}\left(\left\lceil\xi_{2}\right\rceil\right) \cdot v_{2}\left(\left\lceil\xi_{2}\right\rceil\right) \text {, then } \\
& k^{*}=\left\lfloor\xi_{2}\right\rfloor .
\end{aligned}
$$

\section{Conclusions}

In this paper, we introduce an aging effect and a learning effect into the cooperative games on scheduling. Two persons have to cooperate in order to process a set of jobs. The objective is to maximize the multiplication of their rational positive cooperative profits. We show that two scheduling problems $G 2\left|p_{j, r}=P_{0}+a r, d_{j}=d\right| v_{1} v_{2} / L_{\max }$ and $G 2 \mid p_{j, r}=$ $P_{0}-a r, d_{j}=d \mid v_{1} v_{2} / L_{\max }$ are polynomially solvable. The sufficient and necessary conditions for the problems to have positive integer solution are proposed.

For future research, it is interesting to extend the problems to involve other position-dependent processing times scheduling model. Another interesting future research direction is to analyze the problems with other objective functions such as minimizing the number of late jobs, the total weighted completion time, and tardiness.

\section{Conflicts of Interest}

The authors declare that they have no conflicts of interest.

\section{Acknowledgments}

This research is supported by the Key Program of Social Science Foundation of Liaoning Province (Grant no. L15AGL013), the Natural Science Foundation of Liaoning Province (Grant no. 201602545), the National Natural Science Foundation of China (Grant no. 71001074), the Science Research Foundation of Department of Education of Liaoning Province (Grant no. W2015310), and the Humanity and Social Science Research Project of Department of Education of Liaoning Province (Grant no. ZJ2015037).

\section{References}

[1] J. Jin, Y. H. Gu, and G. C. Tang, "Two-person cooperative games on makespan scheduling," Journal of Shanghai Second Polytechnic University, vol. 28, no. 1, pp. 14-17, 2011.

[2] W. Q. Dou, Y. H. Gu, and G. C. Tang, “The Nash bargaining solution of two-person cooperative games on total completion time scheduling," Journal of Chongqing Normal University (Natural Science), vol. 29, no. 5, pp. 1-5, 2012.

[3] Y. H. Gu, J. Jin, and G. C. Tang, "Nash bargaining on maximum flow time scheduling with changeable processing time," Journal of Chongqing Normal University (Natural Science), vol. 29, no. 4, pp. 18-23, 2012.

[4] J. Jin, "Two-person cooperative game problem on scheduling with linear processing time of its starting time," Journal of Nanjing Normal University (Engineering and Technology Edition), vol. 12 , no. 4, pp. 87-92, 2012.

[5] J. Jin, "Two-person cooperative games on total completion time scheduling with changeable processing time," Journal of Suzhou Vocational University, vol. 24, no. 3, pp. 35-39, 2013.
[6] Y. Gu, J. Fan, G. Tang, and J. Zhong, "Maximum latency scheduling problem on two-person cooperative games," Journal of Combinatorial Optimization, vol. 26, no. 1, pp. 71-81, 2013.

[7] L. L. Liu, G. C. Tang, B. Q. Fan, and X. P. Wang, "Twoperson cooperative games on scheduling problems in outpatient pharmacy dispensing process," Journal of Combinatorial Optimization, vol. 30, no. 4, pp. 938-948, 2015.

[8] Q. L. Chen, A new discrete bargaining model on job partition between two manufacturers [Ph.D. thesis], The Chinese University of Hong Kong, 2006.

[9] G. C. Tang, B. Q. Fan, and L. L. Liu, "Classifications, advances and prospects of scheduling games," Journal of Chongqing Normal University (Natural Science), vol. 31, no. 1, pp. 6-14, 2014.

[10] S. A. Gawiejnowicz, "A note on scheduling on a single processor with speed dependent on a number of executed jobs," Information Processing Letters, vol. 57, no. 6, pp. 297-300, 1996.

[11] D. Biskup, "Single-machine scheduling with learning considerations," European Journal of Operational Research, vol. 115, no. 1, pp. 173-178, 1999.

[12] T. C. Cheng and G. Wang, "Single machine scheduling with learning effect considerations," Annals of Operations Research, vol. 98, pp. 273-290, 2000.

[13] G. Mosheiov, "Parallel machine scheduling with a learning effect," Journal of the Operational Research Society, vol. 52, pp. 1165-1169, 2001.

[14] A. Bachman and A. Janiak, "Scheduling jobs with positiondependent processing times," Journal of the Operational Research Society, vol. 55, no. 3, pp. 257-264, 2004.

[15] P. Liu, X. Zhou, and L. Tang, "Two-agent single-machine scheduling with position-dependent processing times," International Journal of Advanced Manufacturing Technology, vol. 48, no. 1-4, pp. 325-331, 2010.

[16] R. A. Rudek, "Scheduling problems with position dependent job processing times: computational complexity results," Annals of Operations Research, vol. 196, pp. 491-516, 2012.

[17] J.-B. Wang and J.-J. Wang, "Flowshop scheduling with a general exponential learning effect," Computers \& Operations Research, vol. 43, pp. 292-308, 2014.

[18] Y. Zhou and Q. Zhang, "Multiple-machine scheduling with learning effects and cooperative games," Mathematical Problems in Engineering, vol. 2015, Article ID 197123, 7 pages, 2015.

[19] X. G. Zhang, Y. Wang, and S. K. Bai, "Single-machine group scheduling problems with deteriorating and learning effect," International Journal of Systems Science, vol. 47, no. 10, pp. 24022410, 2016.

[20] A. Janiak and R. Rudek, "Scheduling jobs under an aging effect," Journal of the Operational Research Society, vol. 61, no. 6, pp. 1041-1048, 2010.

[21] S.-J. Yang, C.-J. Hsu, and D.-L. Yang, "Single-machine scheduling and slack due-date assignment with aging effect and deteriorating maintenance," Optimization Letters, vol. 6, no. 8, pp. 1855-1873, 2012.

[22] R. Rudek, "Minimising maximum lateness in a single machine scheduling problem with processing time-based aging effects," European Journal of Industrial Engineering, vol. 7, no. 2, pp. 206223, 2013.

[23] P. Ji, G. Li, Y. Huo, and J.-B. Wang, "Single-machine common flow allowance scheduling with job-dependent aging effects and a deteriorating maintenance activity," Optimization Letters, vol. 8, no. 4, pp. 1389-1400, 2014. 
[24] J. Y. Choi, "Minimizing total weighted completion time under makespan constraint for two-agent scheduling with jobdependent aging effects," Computers and Industrial Engineering, vol. 83, pp. 237-243, 2015.

[25] X. Zeng, Z. He, H. Yu, and S. Qu, "Bidirectional nonnegative deep model and its optimization in learning," Journal of Optimization, vol. 2016, Article ID 5975120, 8 pages, 2016. 


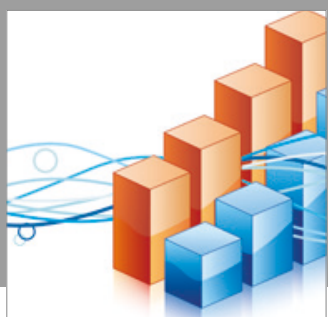

Advances in

Operations Research

vatersals

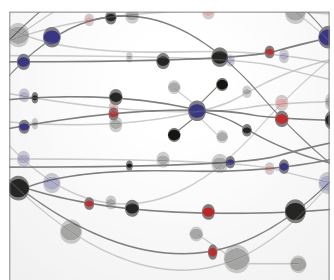

\section{The Scientific} World Journal
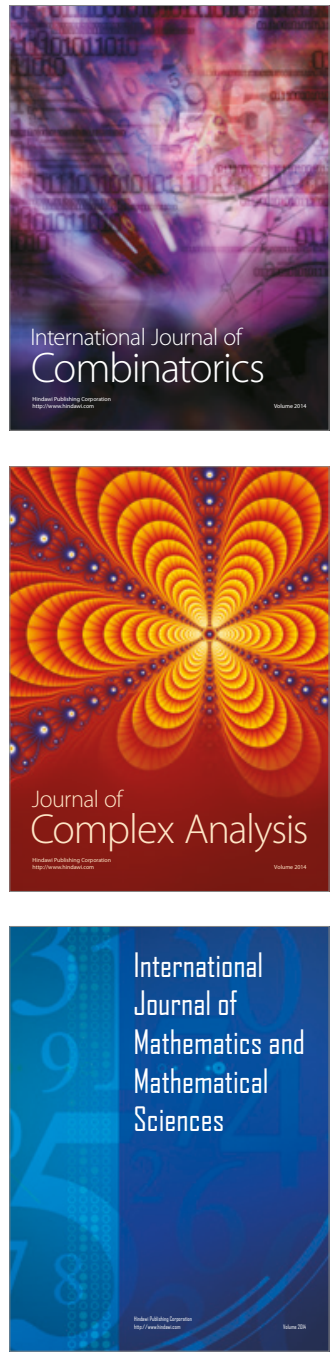
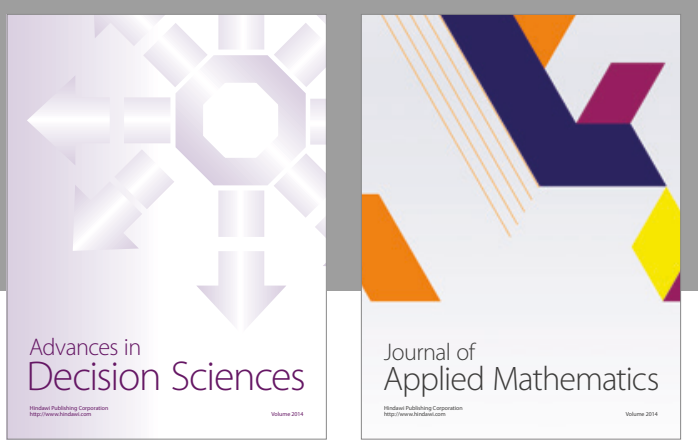

Algebra

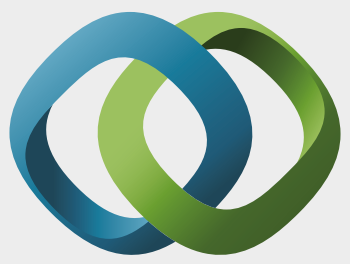

\section{Hindawi}

Submit your manuscripts at

https://www.hindawi.com
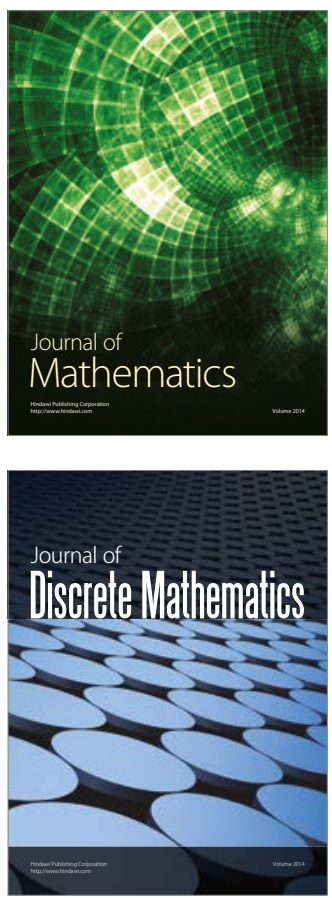

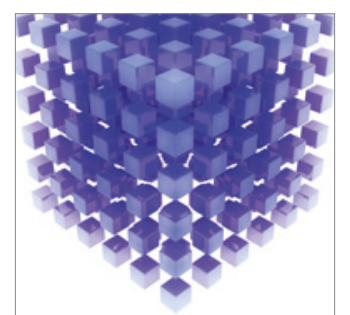

Mathematical Problems in Engineering
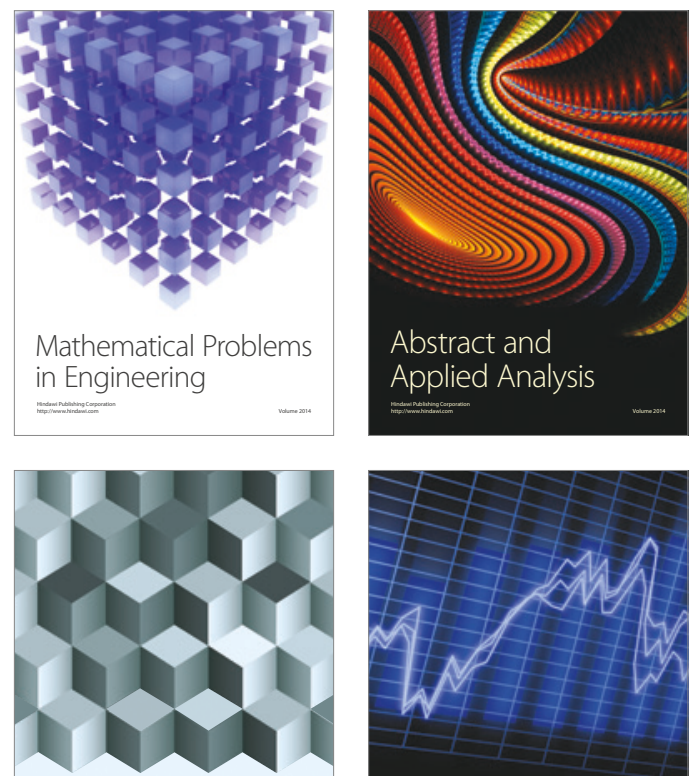

Journal of

Function Spaces

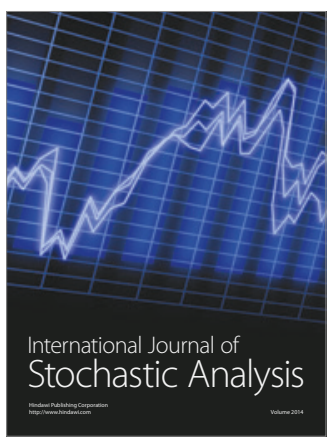

Probability and Statistics
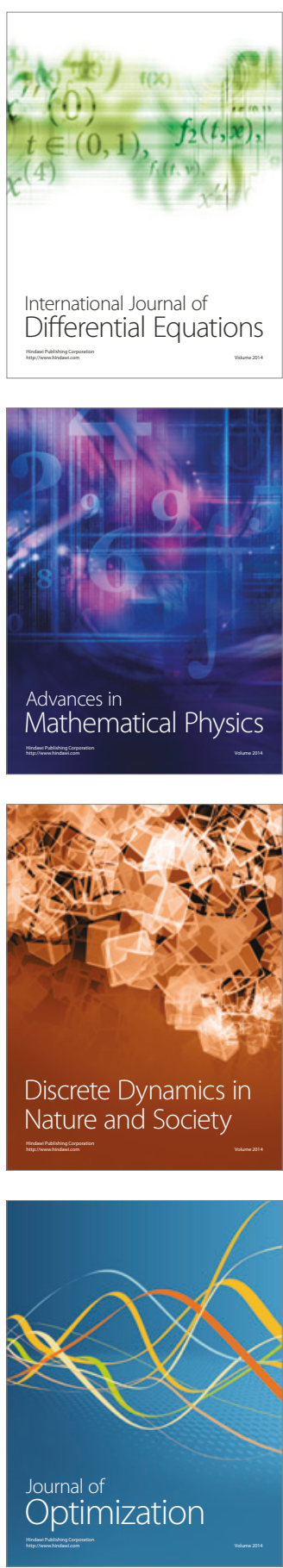